\title{
Pathogenic Reaction of Different Isolates of Colletotrichum capsici on Chilli Cultivars
}

\author{
Rajappa Vithal $^{1}$, Mina D. Koche ${ }^{2^{*}}$, Aparna Tekade ${ }^{1}$ and B. T. Raut ${ }^{1}$ \\ Department of Plant Pathology, Dr. Panjabrao Deshmukh Krishi Vidyapeeth, \\ Akola, Maharashtra, India \\ *Corresponding author
}

\begin{abstract}
A B S T R A C T
The present investigation was aimed to identify the resistant germplasms against Colletotrichum capsici in vitro by artificial inoculation. Ten isolates of Colletotrichum capsici collected from different regions of Vidarbha and Marathwada (Maharashtra), Bidar district (Karnataka) and Guntur district (Andra Pradesh) showing varied type of pathogenic ability against chilli variety Jayanti and designated as strongly, moderately and weakly pathogenic on the basis of per cent leaf area covered by the disease. The varietal screening studies revealed that, remarkable diversity could not observed among the isolates. Strongly pathogenic $\mathrm{Cc}_{6}$ (Guntur) and $\mathrm{Cc}_{9}$ (Akola) recorded the highly susceptible reaction against Jayanti and susceptible reaction against Phule Jyoti, Pusa Jwala, G-4 and $\mathrm{NCH}-162-14-17$ and moderately susceptible reaction against $\mathrm{AKC}-406-1$. Isolate $\mathrm{Cc}_{6}$ exhibited moderately susceptible reaction to AKC-97-20-06-2 and TEJ-F5-01-2-24-14RIII-3-1-6 and susceptible reaction against Hybrid-1, whereas isolate $\mathrm{Cc}_{9}$ exerted susceptible reaction against AKC-97-20-06-2 and TEJ-F5-01-2-24-14-RIII-3-1-6 and highly susceptible reaction against Hybrid-1. Isolate $\mathrm{Cc}_{8}$ (Washim) was exhibited moderately resistant reaction against Phule Jyoti, while isolate $\mathrm{Cc}_{10}$ (Jalna) exerted highly susceptible reaction against AKC-406-1. All other isolates of C. capsici produced varied type of reaction against host varieties, indicating existence of variability among $C$. capsici isolates. Initiation of symptoms, maximum incidence and acervuli production was also comparable among the isolates relates to varied type of aggressiveness.
\end{abstract}

\section{Introduction}

Chilli (Capsicum annuum L.) is one of the most valued spicy vegetable and it has a unique role in human diet. They are nutrient rich with vitamins $\mathrm{A}, \mathrm{C}, \mathrm{E}$ and minerals like pottassium. It is an annual herbaceous vegetable of solanaceae which is cultivated in almost all places. The important chilli growing states are Andhra Pradesh, Karnataka and Tamil Nadu. Inspite of chilli is infected by various biotic and abiotic factors. In biotic several pathogens are causing severe diseases and yield loss. The Chilli anthracnose pathogen $C$. capsici infects diverse host with a high degree of pathogenic variability (Akhtar and Singh, 2007). The genus Colletotrichum causes anthracnose on wide 
range of fruits, vegetables, cereals, grasses and ornamental plants (Dean et al., 2012). The anthracnose is one of serious diseases on chili to cause the yield loss and to reduce the quantity of marketable fruits. In India, in severe cases, pre harvest and post-harvest losses comprise up more than $50 \%$. Significant yield losses were reported from Punjab and Haryana (20-60\%) and Assam (12-30\%) (Sahitya et al., 2014). Disease incidence is recorded from 20 to $80 \%$ on fruits of Capsicum annum and 5 to $20 \%$ on fruits of $C$. frutescens infected in the field conditions. It has been reported that a part of post harvest losses of fruit quality deterioration of chilli is due to anthracnose ranges from 21-47\% (Muthu Kumar and Bhaskaran, 2007). Therefore managing this disease by host plant resistance is a good option. So, the collection of disease samples and to find out the source of resistance among chilli germplasms against fruit rot was carried out. The present investigations were aimed to understand the pathogenic ability and its host plant relationship with each other and differential reaction in chilli.

\section{Materials and Methods}

\section{Collection of disease samples}

The disease samples of fruit rot of chilli (plant parts) were collected from different geographical areas of Vidarbha and Marathwada region of Maharashtra (M.S.), Bidar district of Karnataka and Guntur district of Andhra Pradesh.

\section{Isolation and maintenance of cultures}

The samples showing characteristic symptoms of fruit rot, dieback and anthracnose were collected from different localities and cut along with healthy tissues. The obtained cultures were further purified by single spore isolation technique. The cultures were abbreviated as Cc. (Colletotrichum capsici) and reviewed by periodic transfer and maintained on PDA.

\section{Pathogenicity test}

Monosporous cultures of isolates were obtained from the fungal cultures. The epidermal layer of fruit, leaves of the susceptible variety (Jayanti) of chilli was injured by carborandom powder before inoculation. The spore suspension of each isolate of Colletotrichum capsici was used for inoculating the plants in pots by using sterilized cotton swab. The seedlings were kept for predisposition for 24 hour prior to inoculation by irrigating and covered the moist hesian cloth. Inoculation by cotton swab was made in the evening hour and covered with hesian cloth to provide 100 per cent humidity for spore germination and infection up to 48 hour. Similarly the fruits were also inoculated by smearing the inoculums on the upper surface and incubated in the humid chamber. After inoculating the plants with Colletotrichum capsici, the host plants were examined periodically for development of symptoms. Re-isolations were made and the fungus obtained was compared with the original one.

\section{Screening of chilli germplasms against Colletotrichum capsici}

Four chilli varieties i.e. Jayanti, Phule Jyoti, Pusa Jwala and G-4 procured from Chilli and Vegetable Research Unit, Dr.P.D.K.V. Akola were grown in earthen pot in autoclaved soil. The plants were put to predisposition at age of 60 day for $24 \mathrm{hr}$ prior to inoculation by irrigating and covering with moist hessian cloth. The leaves were washed with distilled water, dried for five minutes. The leaves were again washed with sterile distilled water. Spore suspension of fungal culture grown on petriplates was obtained with standard 
conidial suspension having $10^{6}$ spores per ml. Suspension was smeared on the healthy leaves by slightly injured with carborandum powder rubbing by cotton swab on each leaf. Similarly control was maintained by smearing with sterile distilled water. These plants were covered with moist hesian cloth to provide 100 per cent humidity required for spore germination and development of symptoms. The pots were then kept on net house benches and observed daily for development of symptoms and the disease intensity was recorded after full development of symptoms. The complete experiment was installed in replicated form.

\section{Pathogenic variability by Pinprick method}

Healthy chilli fruits of five germplasms i.e. NCH-162-14-17, AKC-406-1, AKC-97-2006-2, TEJ-F5-01-2-24-14-RIII-3-1-6 and Hybrid-1 were surface sterilized with 0.1 per cent mercuric chloride for 2 minutes and again rinsed with sterile distilled water and incubated at $27 \pm 2^{\circ} \mathrm{C}$ for $48 \mathrm{hr}$ to see the presence of incipient infection. A standard conidial suspension having $10^{6}$ spores per $\mathrm{ml}$ were inoculated by making five pricks per fruits with help of sterilized needle by smearing on each fruit. The inoculated fruits were kept in moist chamber prepared in plastic petriplates by placing double layer of moist blotting sheet at the bottom and incubated at $25 \pm 1^{\circ} \mathrm{C}$ in $\mathrm{BOD}$ incubator.

Chilli fruits were placed on glass rod to avoid contamination on fruit with the blotting sheet. The stalk of the fruit covered with cotton swab dipped in 2 per cent sucrose solution. Observations on the degree of fruit rot were taken on the extent of development of lesions on the fruit up to 15 days after inoculation. The disease intensity on fruits was scored on the basis of scale (Mandal and Beura, 2003) and per cent disease intensity (PDI) and per cent disease incidence was calculated adopting the formula given by Mc Kinney (1923).

\section{Results and Discussion}

\section{Collection, isolation, purification and identification of pathogen}

Fruit rot infected plant parts were collected from different geographical areas of Vidarbha and Marathwada region of Maharashtra, Bidar (Karnataka) and Guntur (Andhra Pradesh). The culture thus obtained was identified as Colletotrichum capsici on the basis of pathogenic ability and morphological characters as per the CMI publications. Purified cultures of the fungus were maintained on PDA slants for further studies and abbreviated as Cc.

\section{Pathogenicity and symptoms}

The characteristic symptoms on leaves are in the form of irregular to circular areas with brown margins. On fruits grayish black colour elongated spots forming acervuli in advanced stage arranged in elliptical manner was examined. The Colletotrichum capsici causing chilli fruit rot was isolated and pathogenicity was proved.

Pathogenic ability of ten isolates of Colletotrichum capsici was tested on a susceptible chilli variety (Jayanti). Observations were recorded on per cent disease intensity after $12 \mathrm{DAI}$ and the results are presented in Table 1 . Two isolates $\mathrm{Cc}_{6}$ and $\mathrm{Cc}_{9}$ were categorized as strongly pathogenic, four $\mathrm{Cc}_{1}, \mathrm{Cc}_{5}, \mathrm{Cc}_{8}$ and $\mathrm{Cc}_{10}$ as moderately pathogenic and rest of the four $\mathrm{Cc}_{2}, \mathrm{Cc}_{3}, \mathrm{Cc}_{4}$ and $\mathrm{Cc}_{7}$ as weakly pathogenic. The basis for categorization of different isolates was adopted by considering the published data of Palarpawar and Ghurde (1994) categorized the isolates as aggressive and less aggressive. Similar observations were also mentioned by 
Sangdee et al., (2011) and Lubna Massodi et al., (2013).

\section{Screening of chilli germplasms against Colletotrichum capsici}

Ten isolates of $C$. capsici were tested against four chilli varieties viz., Jayanti, Phule jyoti, Pusa jwala and G-4 procured from Chilli and
Vegetable Research Unit, Dr.P.D.K.V. Akola. Same isolates were also tested on fruits of five chilli germplasms viz., $\mathrm{NCH}-162-14-17$, AKC-406-1, AKC-97-20-06-2, TEJ-F5-01-224-14-RIII-3-1-6 and Hybrid-1, to assess their pathogenic reaction on the basis of $0-5$ disease grading scale (Mandal and Beura, 2003).

\begin{tabular}{|c|c|c|}
\hline \multicolumn{2}{|c|}{ Disease rating scale $(\mathbf{0}-\mathbf{5})$} & \\
\hline Rating grade & Disease intensity $(\mathbf{\%})$ & Pathogenic Reaction \\
\hline $\mathbf{0}$ & 0 & Highly resistance (HR) \\
\hline $\mathbf{1}$ & $1-5$ & Resistance (R) \\
\hline $\mathbf{2}$ & $6-10$ & Moderately resistance (MR) \\
\hline $\mathbf{3}$ & $11-25$ & Moderately susceptible (MS) \\
\hline $\mathbf{4}$ & $26-50$ & Susceptible (S) \\
\hline $\mathbf{5}$ & $>50$ & Highly susceptible (HS) \\
\hline
\end{tabular}

Jayanti was observed as moderately susceptible against five isolates viz., $\mathrm{Cc}_{2}, \mathrm{Cc}_{3}$, $\mathrm{Cc}_{4}, \mathrm{Cc}_{7}$ and $\mathrm{Cc}_{10}$, while $\mathrm{Cc}_{1}, \mathrm{Cc}_{5}$ and $\mathrm{Cc}_{8}$ exhibited susceptible reaction, whereas $\mathrm{Cc}_{6}$ (Guntur) and $\mathrm{Cc}_{9}$ (Akola) exhibiting more virulence against Jayanti exhibiting highly susceptible reaction. Phule jyoti was moderately resistant to isolate $\mathrm{Cc}_{8}$ (Washim) indicating minimum virulence, while $\mathrm{Cc}_{3}$, $\mathrm{Cc}_{5}, \mathrm{Cc}_{7}$ and $\mathrm{Cc}_{10}$ exerted moderately susceptible reaction and isolate $\mathrm{Cc}_{1}, \mathrm{Cc}_{2}, \mathrm{Cc}_{4}$, $\mathrm{Cc}_{6}$ and $\mathrm{Cc}_{9}$ exhibited the high virulence i.e. susceptible reaction against Phule jyoti indicating variability among the isolates. Variety Pusa jwala exerted moderately susceptible reaction to $\mathrm{Cc}_{1}, \mathrm{Cc}_{2}, \mathrm{Cc}_{3}, \mathrm{Cc}_{4}$ and $\mathrm{Cc}_{7}$, whereas susceptible reaction was observed against $\mathrm{Cc}_{5}, \mathrm{Cc}_{6}, \mathrm{Cc}_{8}, \mathrm{Cc}_{9}$ and $\mathrm{Cc}_{10}$ indicating maximum virulence. Differential reaction were also existed against G-4, wherein $\mathrm{Cc}_{7}$ (Karla) showed moderately susceptible reaction, whereas all other isolates exhibited susceptible reaction showing maximum virulence against G-4 (Table 2).

Table.1 Pathogenic ability of $C$. capsici isolates showing disease reaction against susceptible variety (Jayanti)

\begin{tabular}{|c|l|c|c|}
\hline Isolate & Location & PDI (\%) & Reaction \\
\hline $\mathbf{C c}_{\boldsymbol{1}}$ & Chinchola & 43.33 & MP \\
\hline $\mathbf{C c}_{\mathbf{2}}$ & Parbhani & 19.50 & WP \\
\hline $\mathbf{C c}_{\mathbf{3}}$ & Bidar & 17.67 & WP \\
\hline $\mathbf{C c}_{\mathbf{4}}$ & Divatana & 24.33 & WP \\
\hline $\mathbf{C c}_{\mathbf{5}}$ & Neemkhed & 37.17 & MP \\
\hline $\mathbf{C c}_{\mathbf{6}}$ & Guntur & 51.50 & SP \\
\hline $\mathbf{C c}_{\mathbf{7}}$ & Karla & 18.67 & WP \\
\hline $\mathbf{C c}_{\mathbf{8}}$ & Washim & 33.83 & MP \\
\hline $\mathbf{C c}_{\mathbf{9}}$ & Akola & 51.83 & SP \\
\hline $\mathbf{C c}_{\mathbf{1 0}}$ & Jalna & 38.83 & MP \\
\hline
\end{tabular}


Table.2 Pathogenic reaction of $C$. capsici isolates on leaves of chilli cultivars

\begin{tabular}{|c|c|c|c|c|c|}
\hline \multirow{2}{*}{$\begin{array}{l}\text { Sr. } \\
\text { No. }\end{array}$} & \multirow[t]{2}{*}{ Isolate } & \multicolumn{4}{|c|}{ Per cent disease intensity } \\
\hline & & Jayanti & Phule Jyoti & Pusa Jwala & G - 4 \\
\hline 1 & $\mathrm{Cc}_{1}$ & $\begin{array}{c}43.33 \\
(41.15)\end{array}$ & $\begin{array}{c}36.83 \\
(37.35)\end{array}$ & $\begin{array}{c}24.27 \\
(30.20)\end{array}$ & $\begin{array}{c}40.83 \\
(40.28)\end{array}$ \\
\hline 2 & $\mathrm{Cc}_{2}$ & $\begin{array}{c}19.50 \\
(26.21)\end{array}$ & $\begin{array}{c}33.00 \\
(35.06)\end{array}$ & $\begin{array}{c}18.83 \\
(25.70)\end{array}$ & $\begin{array}{c}45.66 \\
(42.53)\end{array}$ \\
\hline 3 & $\mathrm{Cc}_{3}$ & $\begin{array}{c}17.67 \\
(24.88)\end{array}$ & $\begin{array}{c}23.33 \\
(28.86)\end{array}$ & $\begin{array}{c}16.33 \\
(23.81)\end{array}$ & $\begin{array}{c}28.66 \\
(32.39)\end{array}$ \\
\hline 4 & $\mathrm{Cc}_{4}$ & $\begin{array}{c}24.33 \\
(29.53)\end{array}$ & $\begin{array}{c}35.33 \\
(36.45)\end{array}$ & $\begin{array}{c}21.07 \\
(27.35)\end{array}$ & $\begin{array}{c}26.00 \\
(28.66)\end{array}$ \\
\hline 5 & $\mathrm{Cc}_{5}$ & $\begin{array}{c}37.17 \\
(37.58)\end{array}$ & $\begin{array}{c}12.16 \\
(20.44)\end{array}$ & $\begin{array}{c}30.00 \\
(33.21)\end{array}$ & $\begin{array}{c}33.83 \\
(35.55)\end{array}$ \\
\hline 6 & $\mathrm{Cc}_{6}$ & $\begin{array}{c}51.50 \\
(45.86)\end{array}$ & $\begin{array}{c}26.56 \\
(30.98)\end{array}$ & $\begin{array}{c}31.57 \\
(34.20)\end{array}$ & $\begin{array}{c}31.00 \\
(33.83)\end{array}$ \\
\hline 7 & $\mathrm{Cc}_{7}$ & $\begin{array}{c}18.67 \\
(25.62)\end{array}$ & $\begin{array}{c}18.56 \\
(25.55)\end{array}$ & $\begin{array}{c}23.83 \\
(29.20)\end{array}$ & $\begin{array}{c}23.33 \\
(28.86)\end{array}$ \\
\hline 8 & $\mathrm{Cc}_{8}$ & $\begin{array}{c}33.83 \\
(35.55)\end{array}$ & $\begin{array}{c}8.30 \\
(16.74)\end{array}$ & $\begin{array}{c}31.33 \\
(34.02)\end{array}$ & $\begin{array}{c}33.50 \\
(35.37)\end{array}$ \\
\hline 9 & $\mathrm{Cc}_{9}$ & $\begin{array}{c}51.83 \\
(46.03)\end{array}$ & $\begin{array}{c}30.66 \\
(33.65)\end{array}$ & $\begin{array}{c}34.16 \\
(35.79)\end{array}$ & $\begin{array}{c}30.50 \\
(33.52)\end{array}$ \\
\hline 10 & $\mathrm{Cc}_{10}$ & $\begin{array}{c}38.83 \\
(38.53)\end{array}$ & $\begin{array}{c}25.67 \\
(30.40)\end{array}$ & $\begin{array}{c}33.16 \\
(35.18)\end{array}$ & $\begin{array}{c}29.50 \\
(32.90)\end{array}$ \\
\hline F test & & Sig. & Sig. & Sig. & Sig. \\
\hline SE $(m$ & & 0.27 & 0.48 & 0.49 & 0.52 \\
\hline CD (F & 01) & 1.11 & 1.96 & 1.98 & 2.10 \\
\hline
\end{tabular}

Table.3 Varietal reaction of chilli cultivars against $C$. capsici isolates

\begin{tabular}{|c|l|c|c|c|c|c|c|c|c|c|c|}
\hline Sr. No. & \multirow{2}{*}{ Isolate } & \multicolumn{10}{|c|}{ Reaction } \\
\cline { 3 - 14 } & & $\mathbf{C c}_{\mathbf{1}}$ & $\mathbf{C c}_{\mathbf{2}}$ & $\mathbf{C c}_{\mathbf{3}}$ & $\mathbf{C c}_{\boldsymbol{4}}$ & $\mathbf{C c}_{\mathbf{5}}$ & $\mathbf{C c}_{\mathbf{6}}$ & $\mathbf{C c}_{\mathbf{7}}$ & $\mathbf{C c}_{\mathbf{8}}$ & $\mathbf{C c _ { 9 }}$ & $\mathbf{C c}_{\mathbf{1 0}}$ \\
\hline $\mathbf{1}$ & Jayanti & $\mathrm{S}$ & $\mathrm{MS}$ & $\mathrm{MS}$ & $\mathrm{MS}$ & $\mathrm{S}$ & $\mathrm{HS}$ & $\mathrm{MS}$ & $\mathrm{S}$ & $\mathrm{HS}$ & $\mathrm{S}$ \\
\hline $\mathbf{2}$ & Phule Jyoti & $\mathrm{S}$ & $\mathrm{S}$ & $\mathrm{MS}$ & $\mathrm{S}$ & $\mathrm{MS}$ & $\mathrm{S}$ & $\mathrm{MS}$ & $\mathrm{MR}$ & $\mathrm{S}$ & $\mathrm{MS}$ \\
\hline $\mathbf{3}$ & Pusa Jwala & $\mathrm{MS}$ & $\mathrm{MS}$ & $\mathrm{MS}$ & $\mathrm{MS}$ & $\mathrm{S}$ & $\mathrm{S}$ & $\mathrm{MS}$ & $\mathrm{S}$ & $\mathrm{S}$ & $\mathrm{S}$ \\
\hline $\mathbf{4}$ & $\mathrm{G}-4$ & $\mathrm{~S}$ & $\mathrm{~S}$ & $\mathrm{~S}$ & $\mathrm{~S}$ & $\mathrm{~S}$ & $\mathrm{~S}$ & $\mathrm{MS}$ & $\mathrm{S}$ & $\mathrm{S}$ & $\mathrm{S}$ \\
\hline
\end{tabular}


Table.4 Aggressiveness / virulence of Colletotrichum capsici isolates against chilli germplasm (fruits)

\begin{tabular}{|c|c|c|c|c|c|c|}
\hline Isolates & Varieties & $\begin{array}{l}\text { Hrs to initiate } \\
\text { symptoms }\end{array}$ & $\begin{array}{l}\text { Days for max } \\
\text { symptoms }\end{array}$ & $\begin{array}{c}\text { Number of pricks } \\
\text { infected }\end{array}$ & $\%$ infection & $\begin{array}{l}\text { Days to form } \\
\text { acervuli }\end{array}$ \\
\hline \multirow[t]{5}{*}{$\mathrm{Cc}_{1}$} & NCH-162-14-17 & 72 & 8 & 16 & 80 & 5 \\
\hline & AKC-406-1 & 52 & 6 & 18 & 90 & 3 \\
\hline & AKC-97-20-06-2 & 85 & 8 & 15 & 75 & 6 \\
\hline & TEJ-F5-01-2-24-14-RIII-3-1-6 & 85 & 9 & 13 & 65 & 6 \\
\hline & Hybrid-1 & 52 & 9 & 18 & 90 & 4 \\
\hline \multirow[t]{5}{*}{$\mathrm{Cc}_{2}$} & NCH-162-14-17 & 75 & 8 & 16 & 80 & 5 \\
\hline & AKC-406-1 & 90 & 9 & 13 & 65 & 6 \\
\hline & AKC-97-20-06-2 & 90 & 9 & 16 & 80 & 6 \\
\hline & TEJ-F5-01-2-24-14-RIII-3-1-6 & 75 & 8 & 15 & 75 & 5 \\
\hline & Hybrid-1 & 52 & 6 & 20 & 100 & 3 \\
\hline \multirow[t]{5}{*}{$\mathrm{Cc}_{3}$} & NCH-162-14-17 & 52 & 6 & 20 & 100 & 3 \\
\hline & AKC-406-1 & 95 & 9 & 13 & 65 & 6 \\
\hline & AKC-97-20-06-2 & 96 & 9 & 13 & 65 & 6 \\
\hline & TEJ-F5-01-2-24-14-RIII-3-1-6 & 90 & 9 & 15 & 75 & 6 \\
\hline & Hybrid-1 & 52 & 6 & 20 & 100 & 3 \\
\hline \multirow[t]{5}{*}{$\mathrm{Cc}_{4}$} & NCH-162-14-17 & 74 & 8 & 17 & 85 & 5 \\
\hline & AKC-406-1 & 90 & 8 & 15 & 75 & 6 \\
\hline & AKC-97-20-06-2 & 72 & 8 & 16 & 80 & 5 \\
\hline & TEJ-F5-01-2-24-14-RIII-3-1-6 & 52 & 6 & 18 & 90 & 4 \\
\hline & Hybrid-1 & 52 & 6 & 20 & 100 & 3 \\
\hline \multirow[t]{5}{*}{$\mathrm{Cc}_{5}$} & NCH-162-14-17 & 85 & 8 & 14 & 70 & 6 \\
\hline & AKC-406-1 & 96 & 9 & 9 & 45 & 6 \\
\hline & AKC-97-20-06-2 & 85 & 8 & 15 & 75 & 6 \\
\hline & TEJ-F5-01-2-24-14-RIII-3-1-6 & 52 & 6 & 20 & 100 & 3 \\
\hline & Hybrid-1 & 90 & 9 & 12 & 60 & 6 \\
\hline
\end{tabular}

Note: Four fruits per variety, 5 pricks per fruit 
Aggressiveness / virulence of Colletotrichum capsici isolates against chilli germplasm (fruits) (cont....)

\begin{tabular}{|c|c|c|c|c|c|c|}
\hline Isolates & Varieties & $\begin{array}{c}\text { Hrs to initiate } \\
\text { symptoms }\end{array}$ & $\begin{array}{c}\text { Days for max } \\
\text { symptoms }\end{array}$ & $\begin{array}{c}\text { Number of pricks } \\
\text { infected }\end{array}$ & $\%$ infection & $\begin{array}{c}\text { Days to form } \\
\text { acervuli }\end{array}$ \\
\hline \multirow[t]{5}{*}{$\mathrm{Ce}_{6}$} & NCH-162-14-17 & 72 & 8 & 16 & 80 & 5 \\
\hline & AKC-406-1 & 96 & 9 & 11 & 55 & 6 \\
\hline & AKC-97-20-06-2 & 85 & 9 & 12 & 60 & 6 \\
\hline & TEJ-F5-01-2-24-14-RIII-3-1-6 & 90 & 9 & 11 & 55 & 6 \\
\hline & Hybrid-1 & 72 & 8 & 17 & 85 & 5 \\
\hline \multirow[t]{5}{*}{$\mathrm{Cc}_{7}$} & NCH-162-14-17 & 72 & 8 & 17 & 85 & 5 \\
\hline & AKC-406-1 & 52 & 6 & 18 & 90 & 4 \\
\hline & AKC-97-20-06-2 & 96 & 9 & 12 & 60 & 6 \\
\hline & TEJ-F5-01-2-24-14-RIII-3-1-6 & 52 & 6 & 18 & 90 & 4 \\
\hline & Hybrid-1 & 52 & 6 & 20 & 100 & 3 \\
\hline \multirow[t]{5}{*}{$\mathrm{Cc}_{8}$} & NCH-162-14-17 & 52 & 6 & 20 & 100 & 3 \\
\hline & AKC-406-1 & 90 & 9 & 13 & 65 & 6 \\
\hline & AKC-97-20-06-2 & 52 & 6 & 20 & 100 & 3 \\
\hline & TEJ-F5-01-2-24-14-RIII-3-1-6 & 96 & 9 & 12 & 60 & 6 \\
\hline & Hybrid-1 & 52 & 6 & 20 & 100 & 3 \\
\hline \multirow[t]{5}{*}{$\mathrm{Cc}_{9}$} & NCH-162-14-17 & 72 & 6 & 20 & 100 & 3 \\
\hline & AKC-406-1 & 90 & 8 & 16 & 80 & 6 \\
\hline & AKC-97-20-06-2 & 96 & 8 & 15 & 75 & 6 \\
\hline & TEJ-F5-01-2-24-14-RIII-3-1-6 & 72 & 8 & 17 & 85 & 5 \\
\hline & Hybrid-1 & 52 & 6 & 20 & 100 & 3 \\
\hline \multirow[t]{5}{*}{$\mathrm{Cc}_{10}$} & NCH-162-14-17 & 52 & 6 & 20 & 100 & 3 \\
\hline & AKC-406-1 & 60 & 6 & 20 & 100 & 3 \\
\hline & AKC-97-20-06-2 & 72 & 6 & 19 & 95 & 5 \\
\hline & TEJ-F5-01-2-24-14-RIII-3-1-6 & 72 & 8 & 16 & 80 & 5 \\
\hline & Hybrid-1 & 52 & 6 & 18 & 90 & 4 \\
\hline
\end{tabular}

Note: Four fruits per variety, 5 pricks per fruit 
Table.5 Pathogenic reaction of Colletotrichum capsici isolates against chilli germplasm (fruits)

\begin{tabular}{|c|c|c|c|c|c|c|c|c|c|c|c|}
\hline \multirow{2}{*}{$\begin{array}{l}\text { Sr. } \\
\text { No. }\end{array}$} & \multirow[t]{2}{*}{ Isolate } & \multicolumn{5}{|c|}{ Per cent disease intensity } & \multicolumn{5}{|c|}{ Per cent disease incidence } \\
\hline & & $\begin{array}{c}\text { NCH- } \\
\text { 162-14-17 }\end{array}$ & $\begin{array}{c}\mathrm{AKC}-406- \\
1\end{array}$ & $\begin{array}{l}\text { AKC-97- } \\
20-06--2\end{array}$ & $\begin{array}{c}\text { TEJ-F5-01- } \\
\text { 2-24-14-RIII- } \\
3-1-6\end{array}$ & Hybrid-1 & $\begin{array}{c}\text { NCH- } \\
162-14- \\
17\end{array}$ & $\begin{array}{l}\text { AKC- } \\
406-1\end{array}$ & $\begin{array}{c}\text { AKC-97- } \\
20-06--2\end{array}$ & $\begin{array}{c}\text { TEJ-F5-01- } \\
\text { 2-24-14-RIII- } \\
\text { 3-1-6 }\end{array}$ & $\begin{array}{c}\text { Hybrid } \\
-1\end{array}$ \\
\hline 1 & $\mathrm{Cc}_{1}$ & $\begin{array}{c}26.70 \\
(31.11)\end{array}$ & $\begin{array}{c}43.53 \\
(41.27)\end{array}$ & $\begin{array}{c}33.60 \\
(35.43)\end{array}$ & $\begin{array}{c}20.50 \\
(26.92)\end{array}$ & $\begin{array}{c}50.33 \\
(45.17)\end{array}$ & $\begin{array}{c}86.67 \\
(68.61)\end{array}$ & $\begin{array}{c}86.67 \\
(68.61)\end{array}$ & $\begin{array}{c}86.61 \\
(68.61)\end{array}$ & $\begin{array}{c}66.67 \\
(54.76)\end{array}$ & $\begin{array}{l}100.00 \\
(90.00)\end{array}$ \\
\hline 2 & $\mathrm{Cc}_{2}$ & $\begin{array}{c}30.37 \\
(33.46)\end{array}$ & $\begin{array}{c}19.30 \\
(26.06)\end{array}$ & $\begin{array}{c}38.77 \\
(38.47)\end{array}$ & $\begin{array}{c}34.33 \\
(35.85)\end{array}$ & $\begin{array}{c}43.33 \\
(41.15)\end{array}$ & $\begin{array}{c}86.67 \\
(68.61)\end{array}$ & $\begin{array}{c}66.67 \\
(54.76)\end{array}$ & $\begin{array}{c}86.67 \\
(68.61)\end{array}$ & $\begin{array}{c}80.00 \\
(63.44)\end{array}$ & $\begin{array}{l}100.00 \\
(90.00)\end{array}$ \\
\hline 3 & $\mathrm{Cc}_{3}$ & $\begin{array}{c}34.37 \\
(35.91)\end{array}$ & $\begin{array}{c}18.20 \\
(25.25)\end{array}$ & $\begin{array}{c}33.50 \\
(35.37)\end{array}$ & $\begin{array}{c}49.33 \\
(44.60)\end{array}$ & $\begin{array}{c}51.33 \\
(45.75)\end{array}$ & $\begin{array}{l}100.00 \\
(90.00)\end{array}$ & $\begin{array}{c}53.33 \\
(46.89)\end{array}$ & $\begin{array}{c}73.33 \\
(58.89)\end{array}$ & $\begin{array}{c}80.00 \\
(63.44)\end{array}$ & $\begin{array}{l}100.00 \\
(90.00)\end{array}$ \\
\hline 4 & $\mathrm{Cc}_{4}$ & $\begin{array}{c}18.73 \\
(25.62)\end{array}$ & $\begin{array}{c}42.70 \\
(40.80)\end{array}$ & $\begin{array}{c}27.77 \\
(31.76)\end{array}$ & $\begin{array}{c}35.33 \\
(36.45)\end{array}$ & $\begin{array}{c}48.33 \\
(44.03)\end{array}$ & $\begin{array}{c}86.67 \\
(68.61)\end{array}$ & $\begin{array}{c}86.67 \\
(68.61)\end{array}$ & $\begin{array}{c}73.33 \\
(58.89)\end{array}$ & $\begin{array}{r}100.00 \\
(90.00)\end{array}$ & $\begin{array}{l}100.00 \\
(90.00)\end{array}$ \\
\hline 5 & $\mathrm{Cc}_{5}$ & $\begin{array}{c}31.03 \\
(33.83)\end{array}$ & $\begin{array}{c}19.67 \\
(26.35)\end{array}$ & $\begin{array}{c}33.33 \\
(35.24)\end{array}$ & $\begin{array}{c}43.67 \\
(41.38)\end{array}$ & $\begin{array}{c}34.33 \\
(35.85)\end{array}$ & $\begin{array}{c}73.33 \\
(58.89)\end{array}$ & $\begin{array}{c}46.67 \\
(43.05)\end{array}$ & $\begin{array}{c}80.00 \\
(63.44)\end{array}$ & $\begin{array}{r}100.00 \\
(90.00)\end{array}$ & $\begin{array}{c}60.00 \\
(50.77)\end{array}$ \\
\hline 6 & $\mathrm{Cc}_{6}$ & $\begin{array}{c}43.57 \\
(41.32)\end{array}$ & $\begin{array}{c}23.60 \\
(29.06)\end{array}$ & $\begin{array}{c}19.33 \\
(26.06)\end{array}$ & $\begin{array}{c}23.67 \\
(29.13)\end{array}$ & $\begin{array}{c}30.00 \\
(33.21)\end{array}$ & $\begin{array}{c}80.00 \\
(63.44)\end{array}$ & $\begin{array}{c}46.67 \\
(43.05)\end{array}$ & $\begin{array}{c}60.00 \\
(50.77)\end{array}$ & $\begin{array}{c}60.00 \\
(50.77)\end{array}$ & $\begin{array}{c}86.66 \\
(68.53)\end{array}$ \\
\hline 7 & $\mathrm{Cc}_{7}$ & $\begin{array}{c}16.93 \\
(24.35)\end{array}$ & $\begin{array}{c}33.93 \\
(35.67)\end{array}$ & $\begin{array}{c}18.27 \\
(25.33)\end{array}$ & $\begin{array}{c}38.67 \\
(38.47)\end{array}$ & $\begin{array}{c}49.00 \\
(44.43)\end{array}$ & $\begin{array}{c}80.00 \\
(63.44)\end{array}$ & $\begin{array}{c}93.33 \\
(75.00)\end{array}$ & $\begin{array}{c}46.67 \\
(43.11)\end{array}$ & $\begin{array}{c}86.67 \\
(68.61)\end{array}$ & $\begin{array}{l}100.00 \\
(90.00)\end{array}$ \\
\hline 8 & $\mathrm{Cc}_{8}$ & $\begin{array}{c}38.37 \\
(38.29)\end{array}$ & $\begin{array}{c}28.80 \\
(32.46)\end{array}$ & $\begin{array}{c}37.63 \\
(37.82)\end{array}$ & $\begin{array}{c}18.77 \\
(25.62)\end{array}$ & $\begin{array}{c}48.50 \\
(44.14)\end{array}$ & $\begin{array}{l}100.00 \\
(90.00)\end{array}$ & $\begin{array}{c}60.00 \\
(50.77)\end{array}$ & $\begin{array}{l}100.00 \\
(90.00)\end{array}$ & $\begin{array}{c}46.67 \\
(43.11)\end{array}$ & $\begin{array}{l}100.00 \\
(90.00)\end{array}$ \\
\hline 9 & $\mathrm{Cc}_{9}$ & $\begin{array}{c}28.63 \\
(32.33)\end{array}$ & $\begin{array}{c}18.47 \\
(25.48)\end{array}$ & $\begin{array}{c}33.53 \\
(35.37)\end{array}$ & $\begin{array}{c}39.10 \\
(38.70)\end{array}$ & $\begin{array}{c}54.50 \\
(47.38)\end{array}$ & $\begin{array}{l}100.00 \\
(90.00)\end{array}$ & $\begin{array}{c}80.00 \\
(63.44)\end{array}$ & $\begin{array}{c}66.67 \\
(54.76)\end{array}$ & $\begin{array}{c}93.33 \\
(75.00)\end{array}$ & $\begin{array}{l}100.00 \\
(90.00)\end{array}$ \\
\hline 10 & $\mathrm{Cc}_{10}$ & $\begin{array}{c}43.20 \\
(41.09)\end{array}$ & $\begin{array}{c}58.57 \\
(49.95)\end{array}$ & $\begin{array}{c}40.10 \\
(39.29)\end{array}$ & $\begin{array}{c}28.83 \\
(32.46)\end{array}$ & $\begin{array}{c}45.50 \\
(42.42)\end{array}$ & $\begin{array}{l}100.00 \\
(90.00)\end{array}$ & $\begin{array}{l}100.00 \\
(90.00)\end{array}$ & $\begin{array}{c}93.33 \\
(75.00)\end{array}$ & $\begin{array}{c}93.33 \\
(75.00)\end{array}$ & $\begin{array}{c}86.66 \\
(68.53)\end{array}$ \\
\hline \multicolumn{2}{|c|}{ F test } & Sig. & Sig. & Sig. & Sig. & Sig. & Sig. & Sig. & Sig. & Sig. & Sig. \\
\hline \multicolumn{2}{|c|}{$\mathrm{SE}(\mathrm{m}) \pm$} & 0.39 & 0.30 & 0.32 & 0.28 & 0.31 & 9.97 & 8.15 & 12.13 & 9.95 & 7.92 \\
\hline \multicolumn{2}{|c|}{$\mathrm{CD}(\mathrm{P}=\mathbf{0 . 0 1})$} & 1.58 & 1.22 & 1.30 & 1.13 & 1.25 & 40.13 & 40.13 & 48.83 & 38.05 & 31.88 \\
\hline
\end{tabular}


Table.6 Disease reaction of Colletotrichum capsici isolates against chilli germplasm (fruits)

\begin{tabular}{|c|c|c|c|c|c|c|c|c|c|c|c|}
\hline \multirow{2}{*}{$\begin{array}{l}\text { Sr. } \\
\text { No. }\end{array}$} & \multirow[t]{2}{*}{ Variety } & \multicolumn{10}{|c|}{ Reaction } \\
\hline & & $\mathbf{C c}_{1}$ & $\mathrm{Cc}_{2}$ & $\mathrm{Cc}_{3}$ & $\mathbf{C c}_{4}$ & $\mathrm{Cc}_{5}$ & $\mathrm{Cc}_{6}$ & $\mathbf{C c}_{7}$ & $\mathrm{Cc}_{8}$ & Cc9 & $\mathbf{C c}_{10}$ \\
\hline 1 & $\mathrm{NCH}-162-14-17$ & $\mathrm{~S}$ & $\mathrm{~S}$ & $\mathrm{~S}$ & MS & $\mathrm{S}$ & $\mathrm{S}$ & MS & $\mathrm{S}$ & $\mathrm{S}$ & $\mathrm{S}$ \\
\hline 2 & AKC-406-1 & $S$ & MS & MS & $\mathrm{S}$ & MS & MS & $\mathrm{S}$ & $\mathrm{S}$ & MS & HS \\
\hline 3 & AKC-97-20-06-2 & $\mathrm{S}$ & $\mathrm{S}$ & $\mathrm{S}$ & $\mathrm{S}$ & $\mathrm{S}$ & MS & MS & $\mathrm{S}$ & $\mathrm{S}$ & $\mathrm{S}$ \\
\hline 4 & TEJ-F5-01-2-24-14-RIII-3-1-6 & MS & $\mathrm{S}$ & $\mathrm{S}$ & $\mathrm{S}$ & $\mathrm{S}$ & MS & $\mathrm{S}$ & MS & $\mathrm{S}$ & $\mathrm{S}$ \\
\hline 5 & Hybrid - 1 & HS & $\mathrm{S}$ & HS & $\mathrm{S}$ & $\mathrm{S}$ & $\mathrm{S}$ & $\mathrm{S}$ & $\mathrm{S}$ & HS & $\mathrm{S}$ \\
\hline
\end{tabular}


The pathogenic variability of ten $C$. capsici isolates on fruits of five differentials (germplasms) of chilli by pin pricked method, on the basis of period for initiation of symptoms, days for maximum symptoms, per cent infection and days for development of acervuli. In general chilli Hybrid-1 exhibited initial symptoms after $52 \mathrm{hr}$ by all isolates, except $\mathrm{Cc}_{5}(90 \mathrm{hr})$ and $\mathrm{Cc}_{6}(72 \mathrm{hr})$ might be minimum virulence, while duration required for the complete development of symptoms was ranged between 6 to 9 days, depends on the germplasm and pathogenic ability. Maximum incidence i.e. 100 per cent was achieved by $\mathrm{Cc}_{2}, \mathrm{Cc}_{3}, \mathrm{Cc}_{4}, \mathrm{Cc}_{7}, \mathrm{Cc}_{8}$ and $\mathrm{Cc}_{9}$ against Hybrid-1, whereas 45 per cent incidence against $\mathrm{Cc}_{5}$ was recorded in AKC406-1 followed by 55 per cent in TEJ-F5-012-24-14-RIII-3-1-6 against $\mathrm{Cc}_{6}$. All isolates had an ability to develop acervuli structure within 3 to 6 days, on different germplasm lines. Differences in production of acervuli might be due to isolates and host interaction during the process of pathogenesis also revealed the variation (Table 3 and 4).

Chilli germplasm NCH-162-14-17 was moderately susceptible to isolate $\mathrm{Cc}_{4}$ (Divatana) and $\mathrm{Cc}_{7}$ (Karla), while susceptible to rest of the isolates of $C$. capsici indicating maximum virulence. AKC-406-1 exhibited moderately susceptible reaction to $\mathrm{Cc}_{2}, \mathrm{Cc}_{3}$, $\mathrm{Cc}_{5}, \mathrm{Cc}_{6}$ and $\mathrm{Cc}_{9}$, whereas $\mathrm{Cc}_{1}, \mathrm{Cc}_{4}, \mathrm{Cc}_{7}$ and $\mathrm{Cc}_{8}$ exerted susceptible reaction and only $\mathrm{Cc}_{10}$ (Jalna) showed highly susceptible reaction indicating high degree of virulence. Isolate $\mathrm{Cc}_{6}$ (Guntur) and $\mathrm{Cc}_{7}$ exerted moderately susceptible, while rest of the isolates of $C$. capsici showed susceptible reaction against AKC-97-20-06-2. TEJ-F5-01-2-24-14-RIII-31-6 possesses moderately susceptible reaction against $\mathrm{Cc}_{1}, \mathrm{Cc}_{6}$ and $\mathrm{Cc}_{8}$ (Washim), whereas susceptible reaction was observed in rest of the isolates. Differences in pathogenic ability was also found against Hybrid-1, wherein isolate $\mathrm{Cc}_{2}, \mathrm{Cc}_{4}, \mathrm{Cc}_{5}, \mathrm{Cc}_{6}, \mathrm{Cc}_{7}, \mathrm{Cc}_{8}$ and $\mathrm{Cc}_{10}$ were exerted susceptible reaction, while $\mathrm{Cc}_{1}$ (Chinchola), $\mathrm{Cc}_{3}$ (Bidar) and $\mathrm{Cc}_{9}$ (Akola) were highly aggressive and recorded susceptible reaction indicating high degree of virulence (Table 5 and 6).

Host differential reactions revealed the pathogenic variation among all the isolates of C. capsici on a set of 9 differentials germplasms of chilli. Naik et al., (2003) thirty seven chilli genotypes were screened against C. gloeosporioides and C. capsici in pinprick method of inoculation. The present results corroborates with the findings of Mandeep Kaur et al., (2005) who evaluated severity on seventy one chilli germplasm lines against thirty seven isolates of $C$. capsici and most of the germplasm lines were susceptible to almost all the isolates and only few exhibited resistant or moderately resistant reaction to certain isolates. The results also confirm the findings of Ruchi Garg et al., (2007) who evaluated 13 varieties of chilli against $C$. capsici by using pin pricked method. Gupta $e t$ al., (2018) observed that injury to the fruits favoured the $C$. capsici development on detached fruit surface. Varieties Arka Harita, Classica-152 and Madhurima-148 were showing resistance reaction under two drops of conidial suspension after injury the fruits while EC-341075, Pusa Jwala, Pant C-1, Arka Meghna, LAC-434 and Sonakshi-44 were show moderately resistance reaction as compared to rest of varieties after five, seven and ten days of inoculation. Katediya et al., (2019) evaluated thirty chilli germplasms under field conditions against anthracnose disease of chilli. Among them, three germplasms showed resistant reaction (JDNYC 07-73, JCS 10-98 and JDNYC0970).

\section{Acknowledgement}

Authors are thankful to the Department of Plant Pathology, PGI, Dr. Panjabrao 
Deshmukh Krushi Vidyapeeth Akola for his support and providing necessary facilities to carry out this research work.

\section{References}

Akhtar, J. and Singh, M. K. (2007). Studies on the variability in Colletotrichum capsici causing chilli anthracnose. Indian Phytopath. 60(1): 63-67.

Dean, R., Van, J.A.L., Pretorious, Z.A., Hammond- Kosack, K.E. and Di Pietro, A. (2012). The top 10 fungal pathogens in molecular plant pathology. Molecular Plant Pathology. 13: 414-430.

Gupta, V., Kaur, A., Singh, A., Shekhar, H., Singh, R. and Bobde, A. (2018). Screening of different chilli genotypes against anthracnose disease (Colletotrichum capsci) under controlled condition. Int. J. Curr. Microbiol. App. Sci. 7(3):2328-2334

Katediya, M.A., Jaiman, R. K. and Acharya, S. K. (2019). Survey and screening of germplasm against chilli anthracnose. International J. Chemical Studies. 7(3): 543-545

Lubna, Masoodi., Ali, Anwar., Ahmed, S. and Sofi, T.A. (2013). Cultural, morphological and pathogenic variability in Colletotrichum capsici causing die-back and fruit rot of chilli. Asian J. Plant Pathology, 7: 29-41.

Mandal, S.M.A. and Beura, S. K. (2003). Chemical control of leaf curl, anthracnose and ripe fruit rot in chilli. Indian J. Pl. Protect. 31(1): 137-138.

Mandeep, Kaur., Sharma, O.P. and Sharma, P.N. (2005). Identification of sources of resistance against virulence of
Colletotrichum capsici fruit rot of chilli (Capsicum annuum L.). Indian Phytopath. 58 (1): 232-234.

Mc Kinney, H. (1923). A new system of grading plant diseases. J. Agriculture Res. 26: 196217.

Muthu kumar, A. and Bhaskaran, R. (2007). Tactics to manage disease problems in chilli. Spice India: 24-27.

Naik, M. K., Angadi, H. D., Patil, M.G. and Patil, R.G. (2003). Evaluation of chilli genotypes against anthracnose disease. Veg. Sci. 30 (2): 164-165.

Palarpawar, M.Y. and Ghurde, V.R. (1994). Variability in Colletotrichum curcumae the incitant of leaf spot of turmeric in Vidarbha. Indian Phytopath. 47(2):207210.

Ruchi, Garg., Mishra, P.K., Verma, A., Pandey, K.K. and Rai, B. K. (2007). Morphological and pathogenic characterization of Colletotrichum capsici isolates causing anthracnose of chilli. J. Mycol. Pl. Pathol. 37(3): 594-595.

Sangdee, A., Sachan, S. and Khankhum, S. (2011). Morphological, pathological and molecular variability of Colletotrichum capsici causing anthracnose of chilli in the North-east of Thailand. African J. Microbiology Research. 5(25): 4368-4372.

Sahitya, L.U., Deepthi, S.R., Kasim, P.D., Suneetha, P. and Krishna, M.S. (2014). Anthracnose, a Prevalent Disease in Capsicum. Department of Biotechnology, KL University, India. Acharya N.G. Ranga University, Hyderabad. Research Journal of Pharmaceutical, Biological and Chemical Sciences 5 (3): 1583-1604.

\section{How to cite this article:}

Rajappa Vithal, Mina D. Koche, Aparna Tekade and Raut, B. T. 2020. Pathogenic Reaction of Different Isolates of Colletotrichum capsici on Chilli Cultivars. Int.J.Curr.Microbiol.App.Sci. 9(11): 929-939. doi: https://doi.org/10.20546/ijcmas.2020.911.111 